\title{
Methicillin-resistant Staphylococcus aureus (MRSA) in a tertiary surgical and trauma hospital in Benghazi, Libya
}

\author{
Najat Buzaid ${ }^{1}$, Abdel-Naser Elzouki ${ }^{2}$, Ibrahim Taher $^{3}$, Khalifa Sifaw Ghenghesh ${ }^{4}$ \\ ${ }^{1}$ Department of Medicine, Faculty of Medicine, Garyounis University, Benghazi, Libya \\ ${ }^{2}$ Department of Medicine, Hamad Medical Corporation, Weill Cornell Medical College, Doha, Qatar \\ ${ }^{3}$ Department of Microbiology, Faculty of Medicine, Garyounis University, Benghazi, Libya \\ ${ }^{4}$ Department of Microbiology and Immunology, Faculty of Medicine, Al-Fateh University, Tripoli, Libya
}

\begin{abstract}
Introduction: Methicillin resistant Staphylococcus aureus (MRSA) is a multidrug resistant organism that threatens the continued effectiveness of antibiotics worldwide and causes a threat almost exclusively in hospitals and long-term care settings. This study investigated the prevalence of MRSA strains and their sensitivity patterns against various antibiotics used for treating hospitalized patients in a major tertiary surgical hospital in Benghazi, Libya.

Methodology: We investigated 200 non-duplicate S. aureus strains isolated from different clinical specimens submitted to the Microbiology Laboratory at Aljala Surgical and Trauma Hospital, Benghazi, Libya from April to July 2007. Isolates were tested for methicillin resistance by the oxacillin disc-diffusion assay according to Clinical and Laboratory Standards Institute guidelines. MRSA strains were tested for antimicrobial resistance (i.e., vancomycin, ciprofloxacin, erythromycin, chloramphenicol and fusidic acid) using commercial discs. Information on patient demographics and clinical disease was also collected.

Results: Of the isolates examined 31\% (62/200) were MRSA. No significant differences were observed in the prevalence of MRSA among S. aureus from females or males or from different age groups. Most MRSA were isolated from burns and surgical wound infections. Antibiotic resistance patterns of 62 patients with MRSA to vancomycin, ciprofloxacin, fusidic acid, chloramphenicol and erythromycin were $17.7 \%$, $33.9 \%, 41.9 \%, 38.7 \%$ and $46.8 \%$ of cases, respectively.

Conclusion: MRSA prevalence in our hospital was high and this may be the case for other hospitals in Libya. A sound surveillance program of nosocomial infections is urgently needed to reduce the incidence of infections due to MRSA and other antimicrobial-resistant pathogens in Libyan hospitals.
\end{abstract}

Key words: Staphylococcus aureus; MRSA; tertiary-care hospital; Libya

J Infect Dev Ctries 2011; 5(10):723-726.

(Received 29 October 2010 - Accepted 04 May 2011)

Copyright $(9) 2011$ Buzaid et al. This is an open-access article distributed under the Creative Commons Attribution License, which permits unrestricted use, distribution, and reproduction in any medium, provided the original work is properly cited.

\section{Introduction}

The past few decades have seen an alarming increase in the prevalence of antimicrobial resistant pathogens in serious infections [1,2]. With the discovery of penicillin in 1940 the incidence of bacterial infection decreased worldwide until Staphylococcus aureus (S. aureus) began producing an enzyme, beta-lactamase, that destroys penicillin [3]. Increasing resistance to penicillin has led to the development of semi-synthetic groups of penicillin such as methicillin, that are resistant to many genetic variations of the beta-lactamase enzyme $[3,4]$. For years, infection by $S$. aureus was controlled using methicillin and its analogues. However, in 1961 the first strain of methicillin resistant $S$. aureus (MRSA) was isolated $[1,3,4]$. Since then, MRSA has been found worldwide especially in hospitals and nursing homes $[1,2,5]$. Hospital-acquired infections due to MRSA have been associated with an increase in length of hospital stays, mortality rates, and healthcare costs $[6,7]$.

Data exist for many countries regarding prevalence of MRSA [8]. Although nosocomial infections are associated with considerable morbidity and mortality in developed and developing countries, information regarding such infections in Libya in the international literature is scarce [9]. In addition, reports on the prevalence of MRSA in Libyan hospitals are very few. The aim of the present study was to determine the prevalence of MRSA strains and to investigate the sensitivity pattern of these 
strains against various antibiotics used for treating hospitalized patients in a major tertiary hospital in Libya.

\section{Methodology}

Two hundred non-duplicate $S$. aureus strains isolated from clinical specimens of pus from abscesses, wound swabs, blood, cerebrospinal fluid, central venous line tips, endotracheal tube tips, and urine catheter tips were included in the present study. These isolates were submitted to the Microbiology Laboratory at Aljala Surgical and Trauma Hospital (395 beds), Benghazi (population > 650,000 inhabitants), Libya, from April to July 2007.

Patient history was reviewed from hospital records upon discharge from the hospital; records included patient's age, sex, ward, cause of admission, type of sample (i.e., pus, wound swabs, central venous line tips, endotracheal tube tips, blood cultures, urine catheter tips, etc.), and risk factors for MRSA infection, such as diabetes mellitus, burn, skin and soft tissue infection, chronic orthopedic disease, surgical wound infection, invasive procedures in the intensive care unit (ICU), and extremes of age.

Isolates of $S$. aureus were identified by catalase and tube coagulase tests. Resistance to methicillin was determined by the oxacillin disc-diffusion assay according to the guidelines of the Clinical and Laboratory Standards Institute (CLSI) [10]. Isolates of $S$. aureus resistant to oxacillin were further tested for production of penicillin binding protein $2 \mathrm{a}$ (PBP2a) using a slide latex agglutination test (Oxoid, Basingstoke, UK) and for production of green colonies on chromogenic MRSA ID medium (bioMériueux, Marcy L'Etoile, France) according to the manufacturer's instructions. MRSA strains were further tested for resistance to other antimicrobials using commercial discs (Oxoid); vancomycin (30 $\mu \mathrm{g})$, ciprofloxacin $(5 \mu \mathrm{g})$, erythromycin $(15 \mu \mathrm{g})$, chloramphenicol $(30 \mu \mathrm{g})$ and fusidic acid disc (10 $\mu \mathrm{g}$ ); according to the CLSI guidelines in the same manner used for the oxacillin disc testing. The Epi2000 software (Centers for Disease Control and Prevention, Atlanta, GA, USA) was employed for statistical analysis. $P$-values were calculated using $X^{2}$-test and $P<0.05$ was considered statistically significant.

\section{Results}

Of the 200 S. aureus examined $62(31 \%)$ were MRSA. MRSA was detected in 31.8\% (28/88) and $30.4 \%(34 / 112)$ of $S$. aureus from female and male patients, respectively. In addition, MRSA was found among $26.8 \%$ (11/41), 30.9\% (38/123) and 36.1\% (13/36) of $S$. aureus from patients < 1-19, 20-49 and $>50$ years of age $(P>0.05$ for all comparisons), respectively. MRSA was distributed in most wards of the hospital but a higher prevalence was noted in the burns wards, where approximately two-thirds $(68.4 \%, 13 / 19)$ of the patients with $S$. aureus infection had MRSA. According to the type of infection, it was observed in $20.3 \%(26 / 128)$ of $S$. aureus from patients with skin and soft tissue infections in the form of abscesses, cellulitis and diabetic foot; $54 \%(27 / 50)$ of $S$. aureus from patients with surgical wound infection; $40 \%(6 / 15)$ of $S$. aureus from patients who underwent invasive procedures in the ICU; and in 50\% (2/4) of S. aureus from patients with chronic orthopedic disease in the form of chronic osteomyelitis and septic arthritis.

Based on the disc-diffusion results, the antibiotic resistance pattern of 62 patients with MRSA was as follows: vancomycin resistance was observed in 11 $(17.7 \%)$ cases; ciprofloxacin resistance was observed in $21(33.9 \%)$ cases; chloramphenicol resistance was observed in 24 (38.7\%) cases; fusidic acid resistance was observed in $26(41.9 \%)$ cases; and erythromycin resistance was observed in $29(46.8 \%)$ cases.

\section{Discussion}

In the present investigation MRSA was found in $31 \%$ of $S$. aureus isolates examined. Our findings are in line with previous reports from the Mediterranean region. Borg et al. [11] reported on the prevalence of resistance in clinically relevant pathogens within hospitals in eight southeastern Mediterranean countries. They observed a median of $43 \%$ (range = $10-65 \%$ ) for MRSA within isolates of S. aureus. It should be noted that Libya was not included in the previously mentioned study. However, our findings support the view that the Mediterranean region indeed constitutes a high prevalence region for MRSA [11]. Slightly higher rates than ours for MRSA were reported from some other countries in the Middle East. For instance, MRSA was found in $38 \%(111 / 292)$ of $S$. aureus isolated from clinical specimens in 1998 at King Abdulaziz University Hospital, Jeddah, Saudi Arabia [12].

Although an increase in the prevalence of MRSA was observed to correlate with increased age of patients, the differences in the prevalence rates of MRSA among the three age groups examined in the present study were not statistically significant $(P>$ 0.05). Madani reported that MRSA affected all age 
groups, but almost half (45.9\%) of the patients were in the "extremes of age" group $(<1$ or $>60$ years) [12]. Also, the author observed no preference for any gender. We have also observed no differences and the prevalence rates among $S$. aureus from females and males were $31.8 \%$ and $30.4 \%$, respectively.

MRSA was detected in clinical samples examined from different wards in the present investigation; however, the highest prevalence rate of MRSA was found among $S$. aureus from patients with burns $(68.4 \%)$ and surgical wound infections (54\%). Zorgani et al. [13] reported MRSA in 54.2\% $(65 / 120)$ of $S$. aureus isolated from burn patients in the Burn and Plastic Surgery Center in Tripoli, Libya. Others have reported similar findings [12,14].

MRSA found in this study were generally multidrug resistant with increasing resistance to vancomycin, ciprofloxacin, chloramphenicol, fucidic acid and erythromycin. This observation is in agreement with other studies [15-17]. Vancomycin resistant $S$. aureus was recorded in a $17 \%$ of patients with MRSA, which could be related to a prolonged use of vancomycin on one hand and the possibility of the presence of vancomycin resistant enterococci in the hospital on the other [3,4].

To our knowledge this study is the first to report on the prevalence of MRSA in a tertiary hospital in Libya. The prevalence of MRSA in Aljala Surgical and Trauma Hospital in Benghazi was high and this may be the case for other hospitals in Libya. A recent study that included six different hospitals in Tripoli, Libya, found that $36.8 \%$ of health-care workers were MRSA positive [18]. To reduce the incidence of infections due to MRSA and other antimicrobialresistant pathogens in Libyan hospitals a sound surveillance program of nosocomial infections is urgently needed that include monitoring antibiotic susceptibility patterns of such pathogens and establishing wise antibiotic prescription guidelines.

\section{References}

1. Brown D, Edwards D, Hawkey P, Morrison D, Ridqway G, Towner K, Wren M (2005) Guidelines for the laboratory diagnosis and susceptibility testing of methicillin-resistant Staphylococcus aureus (MRSA). Antimicrob Agents Chemother 65: 1000-1018.

2. Jones RN (2008) Key considerations in the treatment of complicated Staphylococcal infections. Clin Microbiol Infect 14: 3-9.

3. Peacock S (2006) Staphylococcus aureus. In: Gillespie S and Hawkey P, editors). Principal and practice of clinical bacteriology. $2^{\text {nd }}$ ed. England: John Wiley and Sons ltd, 620 p.
4. Lowy F (2005) Staphylococcal infections. In: Fauci A, Braunwarld E, Kasper D, Hauser S, Longo D, Jameson J, editors. Harrison's principles of internal medicine. $16^{\text {th }}$ ed. New York: MacGraw-Hill Companies, 2958 p.

5. Kayser FH Staphylococcus aureus. In: Kayser FH, Bienz K A, Eckert J, Zinkernagel RA, editors. Medical Microbiology. New York: Thieme Stuttgart, 724 p.

6. Shurland S, Zhan M, Bradham DD, Roghmann MC (2007) Comparison of mortality risk associated with bacteremia due to methicillin-resistant and methicillin-susceptible Staphylococcus aureus. Infect Control Hosp Epidemiol 28: 273-279.

7. Selvey LA, Whitby M, Johnson B (2000) Nosocomial methicillin-resistant Staphylococcus aureus bacteremia: is it any worse than nosocomial methicillin-sensitive Staphylococcus aureus bacteremia? Infect Control Hosp Epidemiol 21: 645-648.

8. Grundmann H, Aires-de-Sousa M, Boyce J, Tiemersma E (2006) Emergence and resurgence of methicillin-resistant Staphylococcus aureus as a public-health threat. Lancet 368: 874-85.

9. El-Bouri KW (2009) Clinical microbiology services are essential for diagnosis, treatment and prevention of MRSA and other nosocomial pathogens in Libyan healthcare facilities. Libyan J Med 4: 128-129.

10. Clinical and Laboratory Standards Institute/NCCLS (2005) Performance Standards for Antimicrobial Susceptibility Testing; Fifteenth Informational Supplement. CLSI/ NCCLS documents M100-S15. USA.

11. Borg MA, Scicluna E, Kraker M, Bruinsma N (2006) Antibiotic resistance in the southeastern Mediterranean preliminary results from the ARMed project. Euro Surveill 11: 164-167.

12. Madani TA (2002) Epidemiology and clinical features of methicillin-resistant Staphylococcus aureus (MRSA) at the University Hospital, Jeddah, Saudi Arabia. J KAU:Med Sci 10: 3-12.

13. Zorgani A, Shawerf O, Tawil K, El-Turki E, Ghenghesh KS (2009) Inducible clindamycin resistance among staphylococci isolated from burn patients. Libyan J Med 4: 149-152.

14. Anupurba S, Sen MR, Nath G, Sharma BM, Gulati AK, Mohapatra TM (2003) Prevalence of methicillin resistant staphylococcus aureus in a tertiary referral hospital in eastern Uttar Pradesh. Indian J Med Microbiol 21: 49-51.

15. Schito GC (2002) Is antimicrobial resistance also subject to globalization? Clin Microbiol Infect 8: 1-8.

16. Michel M and Gutmann L (1997) Methicillin-resistant Staphylococcus aureus and vancomycin-resistant enterococci: Therapeutic realities and possibilities. Lancet 349: 1901.

17. Markowitz N, Quinn EL, Saravolatz, LD (1992) Trimethoprim-sulfamethoxazole compared with vancomycin for the treatment of Staphylococcus aureus infection. Ann Intern Med 117: 390.

18. Zorgani A, Elahmer O, Franka E, Grera A, Abudher A, Ghenghesh KS (2009) Detection of methicillin-resistant Staphylococcus aureus among healthcare workers in Libyan hospitals. J Hosp Infect 73: 91-92. 


\section{Corresponding author}

Abdel-Naser Elzouki, MBChB, MSc, MD, PhD, FRCP (UK)

Professor and Sr. Consultant of Clinical Medicine

Department of Medicine, Hamad Medical Corporation

Weill Cornell Medical College

PO Box 3050

Doha, Qatar

Telephone: (+974) 66022836

Fax: (+974) 44392489

Email: nelzouki_1999@yahoo.com; aelzouki@hmc.org.qa

Conflict of interests: No conflict of interests is declared. 